\title{
A new method for suppressing excited-state contaminations on the nucleon form factors
}

\author{
Harvey B. Meyer, Konstantin Ottnad and Tobias Schulz* \\ PRISMA Cluster of Excellence, Institut für Kernphysik and Helmholtz Institut Mainz, \\ Johannes Gutenberg-Universität Mainz, 55099 Mainz, Germany \\ E-mail: meyerh@uni-mainz.de, kottnad@uni-mainz.de, \\ schulzt@uni-mainz.de
}

\begin{abstract}
One of the most challenging tasks in lattice calculations of baryon form factors is the analysis and control of excited-state contaminations. Taking the isovector axial form factors of the nucleon as an example, both a dispersive representation and a calculation in chiral effective field theory show that the excited-state contributions become dominant at fixed source-sink separation when the axial current is spatially distant from the nucleon source location. We address this effect with a new method in which the axial current is localized by a Gaussian wave-packet and apply it on a CLS ensemble with $N_{f}=2+1$ flavors of $\mathrm{O}(a)$ improved Wilson fermions with a pion mass of $m_{\pi}=200 \mathrm{MeV}$.
\end{abstract}

The 36th Annual International Symposium on Lattice Field Theory - LATTICE2018 22-28 July, 2018

Michigan State University, East Lansing, Michigan, USA.

\footnotetext{
* Speaker.
} 


\section{Introduction}

The study of the electroweak form factors of the nucleon reveals insights into its internal structure. Electron scattering and atomic spectroscopy can be used to probe the electromagnetic form factors $G_{E}\left(q^{2}\right), G_{M}\left(q^{2}\right)$, whereas for the study of the axial form factors $G_{A}\left(q^{2}\right), G_{P}\left(q^{2}\right)$, which are far less well known than the electromagnetic form factors [1], one has to rely on weak probes, i.e. neutrino scattering or muon capture processes. Although there is a formal description of the strong interaction given by the theory of Quantum chromodynamics (QCD), it remains highly challenging to understand the details of low-energy phenomena of hadronic bound states such as the proton. To this day, many aspects about the distribution of the proton charge and spin as well as the contribution of the gluons to the proton spin are not understood at a satisfactory level.

Phenomenologically, the nucleon axial charge $g_{A}=G_{A}(0)$ is known to two parts per mille from neutron beta decay. In comparison, lattice QCD studies of this low-energy observable still suffer from large statistical and systematic uncertainties [2]. One of the main sources for the latter is the contamination of the relevant correlation functions from excited-state contributions. Here we investigate a new method to overcome this problem by localizing the axial-vector current in position space.

\section{Formalism of nucleon correlation functions}

We start with the Euclidean nucleon two- and three-point correlation functions, given by

$$
\begin{aligned}
C_{2}\left(\vec{p}, t_{s}\right) & =a^{3} \sum_{\vec{x}} e^{-i \vec{p} \cdot \vec{x}} \Gamma_{\beta \alpha}\left\langle\mathscr{N}_{\alpha}\left(\vec{x}, t_{s}\right) \overline{\mathscr{N}}_{\beta}(0)\right\rangle, \\
C_{3, \mathscr{O}}\left(\vec{p}, \vec{p}^{\prime}, t, t_{s}\right) & =a^{6} \sum_{\vec{x}, \vec{y}} e^{i\left(\vec{p}^{\prime}-\vec{p}\right) \cdot \vec{y}} e^{-i \vec{p}^{\prime} \cdot \vec{x}} \Gamma_{\beta \alpha}\left\langle\mathscr{N}_{\alpha}\left(\vec{x}, t_{s}\right) \mathscr{O}(\vec{y}, t) \overline{\mathscr{N}}_{\beta}(0)\right\rangle .
\end{aligned}
$$

The source-sink separation $t_{s}$ measures the Euclidean time between the nucleon creation (source) and annihilation (sink) point. A local operator is inserted at space-time position $y=(\vec{y}, t), 0 \leq$ $t \leq t_{s}$, which has a nucleon matrix element given by $\left\langle N, \vec{p}^{\prime}, s^{\prime}|\mathscr{O}(0)| N, \vec{p}, s\right\rangle=\bar{u}^{s^{\prime}}\left(\vec{p}^{\prime}\right) \mathscr{O}(P, Q) u^{s}(\vec{p})$, $P \equiv p^{\prime}+p, Q \equiv p^{\prime}-p$. Taking the limit $t,\left(t_{s}-t\right) \gg m_{\pi}^{-1}$ and performing a spectral decomposition, one obtains (NB: we use Euclidean Dirac matrices, $\left\{\gamma_{\mu}, \gamma_{v}\right\}=2 \delta_{\mu v}$ )

$$
\begin{aligned}
C_{2}(\vec{p}, t) & =e^{-E_{\vec{p}} t}|Z(\vec{p})|^{2}\left(1+\frac{m_{N}}{E_{\vec{p}}}\right)+\ldots \\
C_{3, \mathscr{O}}\left(\vec{p}, \vec{p}^{\prime}, t, t_{s}\right) & =\frac{Z\left(\vec{p}^{\prime}\right) Z^{*}(\vec{p})}{4 E_{\vec{p}} E_{\vec{p}^{\prime}}} e^{-E_{\vec{p}^{\prime}}\left(t_{s}-t\right)} e^{-E_{\vec{p}} t} \operatorname{Tr}\left(\Gamma\left(-i \not p^{\prime}+m\right) \mathscr{O}(P, Q)(-i \not p+m)\right)+\ldots,
\end{aligned}
$$

where the dots stand for excited-state contributions and $p_{0}=i E_{\vec{p}}=i \sqrt{\vec{p}^{2}+m_{N}^{2}}, p_{0}^{\prime}=i E_{\vec{p}^{\prime}}$.

It is interesting to compare the expression

$$
\hat{C}_{3, \mathscr{O}}\left(\vec{p}, \vec{p}^{\prime}, t, t_{s}\right) \equiv Z\left(\vec{p}^{\prime}\right) Z^{*}(\vec{p}) \int_{-\infty}^{\infty} \frac{d p_{0}}{2 \pi} \int_{-\infty}^{\infty} \frac{d p_{0}^{\prime}}{2 \pi} \operatorname{Tr}\left\{\Gamma \frac{-i \not p^{\prime}+m}{p^{\prime 2}+m^{2}} \mathscr{O}(P, Q) \frac{-i \not p+m}{p^{2}+m^{2}}\right\} e^{i p_{0}^{\prime}\left(t_{s}-t\right)+i p_{0} t}
$$

to Eq. (2.4). Performing the $p_{0}, p_{0}^{\prime}$ integrals by contour integration, the function $\hat{C}_{3, \mathscr{O}}$ is found to contain the ground-state contribution of $C_{3, \mathscr{C}}$, but it also contains additional terms due to the 
singularities of the form factor in the complex plane. What makes $\hat{C}_{3, \mathscr{O}}$ appealing is that, for Lorentz-covariant interpolating operators $(Z(\vec{p})$ independent of $\vec{p})$, it has a manifestly Lorentzcovariant form, which the ground-state contribution in (2.4) does not ${ }^{1}$. Thus it appears that $\hat{C}_{3, \mathscr{O}}$ is a Lorentz-covariant completion of the ground-state contribution to $C_{3, \mathscr{O}}$.

Consider then the case of $\mathscr{O}=A_{\mu}^{a}=\bar{\psi} \gamma_{\mu} \gamma_{5} \frac{\tau^{a}}{2} \psi$ the isovector axial current, for which $\mathscr{O}(P, Q)=$ $\left[\gamma_{\mu} \gamma_{5} G_{A}\left(-Q^{2}\right)-i \gamma_{5} \frac{Q_{\mu}}{2 m_{N}} G_{P}\left(-Q^{2}\right)\right] \frac{\tau^{a}}{2}$ is parametrized by the axial and induced-pseudoscalar form factors, and does not depend on $P$. To exhibit the singularities of the form factors, we use their dispersive representation,

$$
G_{A, P}\left(-Q^{2}\right)=\int_{s_{0}}^{\infty} \frac{d s}{\pi} \frac{\operatorname{Im} G_{A, P}(s)}{s+Q^{2}} .
$$

Inserting these expressions into Eq. (2.5), the additional terms generated can be analyzed more explicitly. In the present case, the induced pseudoscalar form factor contains a pole at $s_{0}=m_{\pi}^{2}$, while the axial current has a three-pion threshold at $s_{0}=9 m_{\pi}^{2}$. Keeping only the pion-pole contribution to the three-point function,

$$
\operatorname{Im} G_{P}(s)=4 \pi M_{N}^{2} g_{A}^{\pi} \delta\left(s-m_{\pi}^{2}\right)+\ldots,
$$

we obtain for the case of $\mathscr{O}=A_{0}^{3}$ in the proton with $\vec{p}^{\prime}=0$ and $\Gamma=\frac{1}{2}\left(1+\gamma_{0}\right)\left(1+i \gamma_{5} \vec{s} \cdot \vec{\gamma}\right)$,

$$
\begin{aligned}
& \hat{C}_{3, A_{0}}\left(-\vec{q}, \overrightarrow{0}, t, t_{s}\right)_{\pi \text { pole }}=\frac{Z(\overrightarrow{0}) Z^{*}(-\vec{q}) M_{N} g_{A}^{\pi} \vec{s} \cdot \vec{q}}{E_{\vec{q}}} \\
& {\left[\frac{2 E_{\vec{q}} e^{-M_{N} t_{s}-\omega_{\vec{q}} t}}{\left(M_{N}+\omega_{\vec{q}}\right)^{2}-E_{\vec{q}}^{2}}-\frac{e^{-E_{\vec{q}} t_{s}-\omega_{\vec{q}}\left(t_{s}-t\right)}}{\omega_{\vec{q}}+E_{\vec{q}}-M_{N}}+\frac{2\left(M_{N}-E_{\vec{q}}\right) e^{-E_{\vec{q}} t-M_{N}\left(t_{s}-t\right)}}{\omega_{\vec{q}}^{2}-\left(E_{\vec{q}}-M_{N}\right)^{2}}\right] .}
\end{aligned}
$$

From the time-dependence of the last term, one identifies it with the ground-state contribution; its denominator is nothing but $Q^{2}+m_{\pi}^{2}$, thus corresponding to the pion-pole contribution to $G_{\mathrm{P}}$. The other terms are subdominant for large $t, t_{s}-t$; they represent $N \pi$ excited-state contributions.

In order to confirm this interpretation, one may calculate the pion-exchange contribution to $C_{3, A_{\mu}}\left(\vec{p}, \vec{p}^{\prime}, t, t_{s}\right)$ in chiral effective theory (ChEFT), using the representation $A_{\mu}^{a}=-2 i f \partial_{\mu} \pi^{a}+\ldots$, with $f$ the pion decay constant, and the interaction Lagrangian

$$
\mathscr{L}_{\text {int }}=\frac{i g_{A}}{f} \bar{\Psi}(x) \gamma_{\mu} \gamma_{5} \frac{\tau^{a}}{2} \Psi(x) \partial_{\mu} \pi^{a}(x)
$$

A straightforward field-theoretic calculation then gives

$$
\begin{aligned}
& C_{3, A_{0}}\left(-\vec{q}, \overrightarrow{0}, t, t_{s}\right)_{\text {ChEFT }, \pi \text { exchange }}=\frac{|Z|^{2} g_{A} \vec{s} \cdot \vec{q}}{2 E_{\vec{q}}} \\
& {\left[\frac{4 E_{\vec{q}}\left(M_{N}+\omega_{\vec{q}}\right) e^{-M_{N} t_{s}-\omega_{\vec{q}} t}}{\left(M_{N}+\omega_{\vec{q}}\right)^{2}-E_{\vec{q}}^{2}}-\frac{\left(\omega_{\vec{q}}+E_{\vec{q}}+M_{N}\right) e^{-E_{\vec{q}} t_{s}-\omega_{\vec{q}}\left(t_{s}-t\right)}}{\omega_{\vec{q}}+E_{\vec{q}}-M_{N}}+\frac{4 M_{N}\left(M_{N}-E_{\vec{q}}\right) e^{-E_{\vec{q}} t-M_{N}\left(t_{s}-t\right)}}{\omega_{\vec{q}}^{2}-\left(E_{\vec{q}}-M_{N}\right)^{2}}\right] .}
\end{aligned}
$$

The last term in the square bracket corresponds to the ground-state contribution and matches exactly the third term in Eq. (2.8) if we identify $g_{A}^{\pi}=g_{A}$. The first and second term of Eqs. (2.8) and (2.10)

\footnotetext{
${ }^{1}$ This observation is most easily made by Fourier-transforming $\hat{C}_{3, \mathscr{O}}$ with respect to $\vec{p}$ and $\vec{p}^{\prime}$ to obtain the coordinate-space three-point function.
} 
also agree in the limit of small $m_{\pi},|\vec{q}|$. In the ChEFT calculation ${ }^{2}$, it is clear that these terms correspond to excited-state contributions. We conclude that $\hat{C}_{3, \mathscr{O}}$ can be helpful in describing the pre-asymptotic form of the three-point function, especially when a pole contribution is present. Importantly, it does not involve additional parameters as compared to the expression of the groundstate contribution, as it is determined by the same form factor.

\subsection{Current insertion in coordinate-space}

Performing a Fourier transform of equation (2.10) to localize the axial current at point $(t, \vec{y})$ in coordinate space yields the following expression,

$$
\int \frac{d^{3} p}{(2 \pi)^{3}} e^{i \vec{p} \cdot \vec{y}} C_{3, A_{0}}\left(\vec{p}, \overrightarrow{0}, t, t_{s}\right)_{\pi \text { exch. }}^{\mathrm{ChEFT}} \approx 2 i|Z|^{2} g_{A} e^{-m_{N} t_{s}} \vec{s} \cdot \overrightarrow{\nabla_{\vec{y}}}\left[G_{\pi}(t, \vec{y})-G_{\pi}\left(t_{s}-t, \vec{y}\right)+\frac{m_{\pi}^{2}}{2 M_{N}} \frac{e^{-m_{\pi}|\vec{y}|}}{4 \pi|\vec{y}|}\right],
$$

where $G_{\pi}(y)=\frac{m_{\pi} K_{1}(m|y|)}{4 \pi^{2}|y|}$ is the pion propagator. We have assumed the axial current to be distant from the origin $|\vec{y}| \gtrsim m_{\pi}^{-1}$, as well as $m_{\pi} \ll M_{N}, m_{\pi}^{2} t_{s} /\left(2 M_{N}\right) \ll 1$. Thus for $|\vec{y}| \gg t, t_{s}-t$, there is a regime where the first two (excited-state) terms actually dominate over the last (ground-state) term.

The observation above motivates the idea of restricting the location of the axial current to the region $|\vec{y}| \lesssim \max \left(s_{0}^{-1 / 2}\right)$ via a 'wave packet'. An important point is that the wave-packet method does not spoil the first-principles nature of the lattice calculation: in a finite interval of $Q^{2}$, the form factor can be parametrized in a systematically improvable way via conformal-mapping techniques. The idea of the wave-packet method is to directly fit this parametrization to the three-point function with a spatially localized (axial, vector, ... ) current. Because we want to determine the form factor primarily at low $Q^{2}$, we should not use an $\vec{x}$-space wave packet with a sharp edge, which will contain high-momentum modes. A Gaussian wave-packet is then the obvious choice.

A further motivation to use a wave-packet arises for flavor singlet currents, for which the signal-to-noise ratio is expected to be improved, since disconnected diagrams contribute fluctuations that do not fall off with the spatial distance (see e.g. $[4,5])$.

\subsection{Wave Packet Method}

In the following, we describe how the wave-packet method can be applied to calculate the axial form factor at low momentum transfer. We introduce the $\left(\vec{p}^{\prime}=0\right)$ nucleon 3-point correlation function with a localized axial current by

$$
C_{3, \vec{s} \cdot \vec{A}}[\psi]\left(t_{s}, t\right)=a^{6} L^{3} \sum_{\vec{x}, \vec{y}} \psi(\vec{y}) \Gamma_{\beta \alpha}\left\langle\mathscr{N}_{\alpha}(x)(\vec{s} \cdot \vec{A}(t, \vec{y})) \overline{\mathscr{N}}_{\beta}(0)\right\rangle=\sum_{\vec{q}} \tilde{\psi}(\vec{q}) C_{3, \vec{s} \cdot \vec{A}}\left(-\vec{q}, \overrightarrow{0}, t, t_{s}\right)
$$

On the torus, the wave packet is given by $\psi(\vec{y})=\frac{1}{L^{3}} \sum_{\vec{q}} e^{i \vec{q} \cdot \vec{y}} \tilde{\psi}(\vec{q})$. We construct a Gaussian wave packet in the plane orthogonal to the nucleon spin by selecting $\tilde{\psi}(\vec{q})=\delta_{\vec{s} \cdot \vec{q}, 0} \exp \left(-\vec{q}_{\perp}^{2} /\left(2 \Delta^{2}\right)\right)$, and

\footnotetext{
${ }^{2}$ See [3] for a complete leading-order calculation in ChEFT; the pion-exchange contribution is found to be dominant.
} 
maintain exact projection onto zero momentum along the spin direction, in order to be sensitive to $G_{\mathrm{A}}$ and not $G_{\mathrm{P}}$. We will focus on the following ratio of 3-point and 2-point correlation functions,

$$
w\left(\Delta, t, t_{s}\right) \equiv \frac{\operatorname{Im} C_{3, \vec{s} \cdot \vec{A}}[\psi]\left(t_{s}, t\right)}{C_{2}\left(\overrightarrow{0}, t_{s}\right)}=\frac{1}{2} \sum_{\vec{q} \in \mathscr{P}_{\perp}} \frac{Z^{*}(-\vec{q})}{Z^{*}(\overrightarrow{0})}\left[1+\frac{m_{N}}{E_{\vec{q}}}\right] G_{A}\left(-Q^{2}\right) e^{-\frac{\vec{q}^{2}}{2 \Delta^{2}}-\left(E_{\vec{q}}-m_{N}\right) t}+\ldots,
$$

where $\mathscr{P}_{\perp}=\{\vec{q} \mid \vec{s} \cdot \vec{q}=0\}$ is the plane transverse to the nucleon spin. In (2.13), the ground-state, $t_{s}$-independent contribution is given explicitly and the dots stand for excited-state contributions. In this exploratory study, we use the dipole parametrization $G_{A}\left(-Q^{2}\right)=g_{A} /\left(1+Q^{2} / M_{A}^{2}\right)^{2}$ of the form factor, but intend to use systematically improvable parametrizations in the near future. Also, we fit only the ground-state contribution to the lattice data; we have not yet attempted to fit the form (2.5) or to include explicitly excited-state terms in the fit ansatz. Thus the goal is to determine the parameters $\left(g_{A}, M_{A}\right)$. We pre-determine the overlap factors $Z(\vec{p})$ from correlated fits to the nucleon two-point correlation functions.

\section{Lattice implementation and results}

We perform an analysis of lattice data obtained in $N_{\mathrm{f}}=2+1 \mathrm{QCD}$ with an $\mathscr{O}(a)$-improved Wilson fermion action [6] and a tree-level improved Lüscher-Weisz gauge action as well as open boundary conditions in the time direction [7]. The nucleon correlation functions were computed on the ensemble D200, created as part of the CLS ("Coordinated Lattice Simulations") initiative. The parameters of the ensemble are given in Table 1. Our observable is fully $\mathrm{O}(a)$ improved.

\begin{tabular}{|c|c|c|c|c|c|c|c|}
\hline$\beta$ & $a / \mathrm{fm}$ & $L / a$ & $L / \mathrm{fm}$ & $T / a$ & $m_{\pi} / \mathrm{MeV}$ & $N_{\text {conf }}$ & $N_{\vec{q}}$ \\
\hline 3.55 & 0.06440 & 64 & 4.1216 & 128 & 200 & 1021 & 179 \\
\hline
\end{tabular}

Table 1: Simulation parameters of the D200 ensemble. The lattice spacing is taken from [8].

We truncate the sum over $\vec{q}$ in Eq. (2.12) to the $N_{\vec{q}}=179$ lowest lattice momenta $\vec{q}=(2 \pi / L) \vec{n}$, $\vec{n} \in \mathbb{Z}^{3}$. The momentum $\vec{p}^{\prime}$ of the nucleon at the sink being set to zero, the momentum transfer is $Q^{2}=2 m_{N}\left(E_{\vec{q}}-m_{N}\right)$. As for the calculation of the gauge expectation values, we use the truncated solver method with bias correction $[9,10]$.

Figure 1 displays the first results for two different spatial localizations $\Delta=0.5 \pi / L$ and $\Delta=$ $3 \pi / L$. In the left panel, a trend of $w\left(\Delta, t, t_{s}\right)$ as a function of $t_{s}$ is seen. For the stronger localization, $w\left(\Delta, t, t_{s}\right)$ is independent of $t_{s}$ for $t \gtrsim 0.4 \mathrm{fm}$, within the statistical uncertainty. In Figure 2 we show the results of correlated two-parameter fits for the axial charge $g_{A}$ and the axial mass $M_{A}$ for $\Delta \in\{3 / 2,5 / 2\} \pi / L$. We used six time slices for the fits $t \in[9,14] a$. In the case displayed in the left panel, the fit has no sensitivity to $M_{A}$, because the wave packet suppresses large-momentum contributions. For stronger localizations (right panel), we are sensitive to both parameters.

Figure 3 shows the fit results for different sizes $\Delta$ of localizations. The smaller source-sink separations show smaller statistical errors but a larger sensitivity of $g_{A}$ to the width of the wave packet. The results for $g_{A}$ are compatible with the result $\left(g_{A}=1.188 \pm 0.025\right)$ obtained from a different method, namely simultaneous fits to nucleon three-point functions at $\vec{q}=0$ with different currents [11], but have a larger statistical error, as they are obtained from a small number of data. 

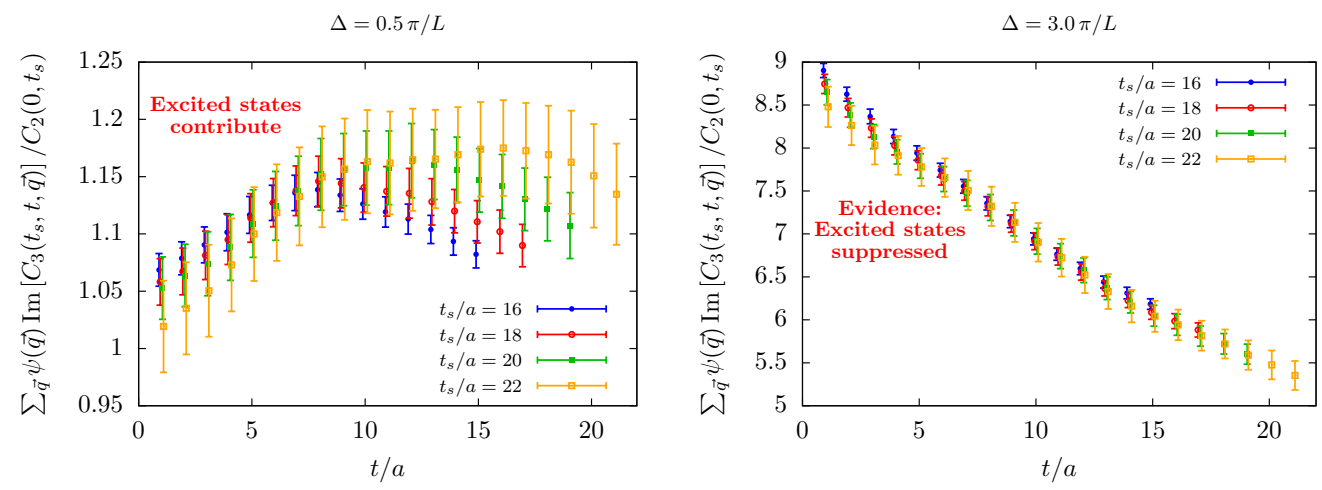

Figure 1: The observable $w\left(\Delta, t, t_{S}\right)$ on ensemble D200, as a function of the current insertion time $t$ for two different momentum-space widths $\Delta \in\{1 / 2,3\} \pi / L$ of the wave packet.
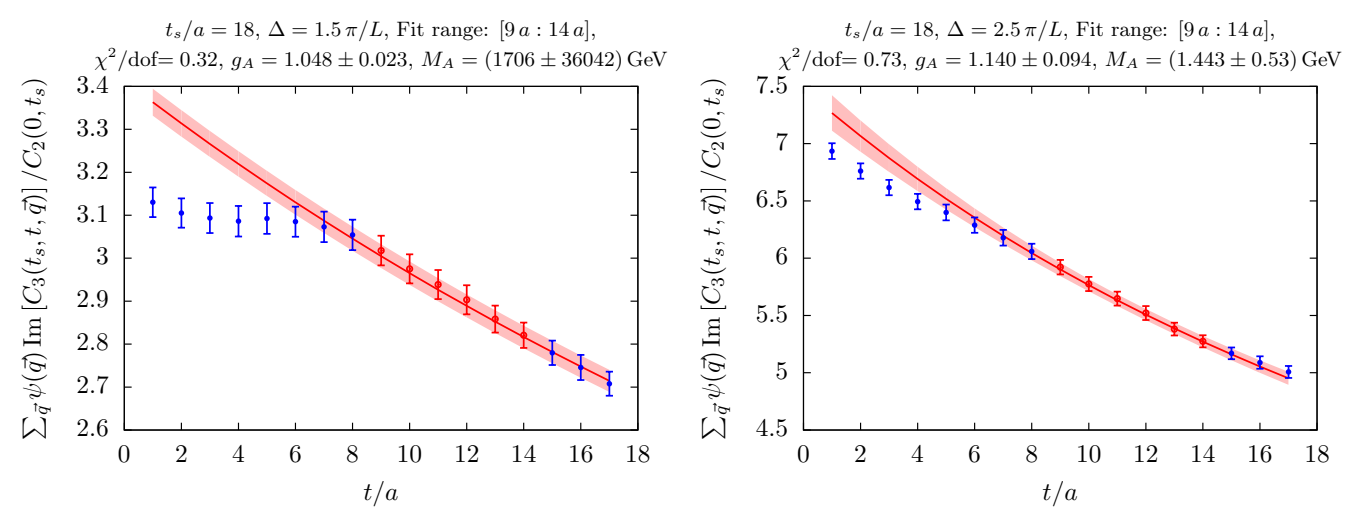

Figure 2: Correlated two-parameter fits to the lattice data for the parameters $\left(g_{A}, M_{A}\right)$, using the ground-state contribution to $w\left(\Delta, t, t_{s}\right)$ given in Eq. (2.13), for $\Delta=1.5 \pi / L$ (left) and $\Delta=2.5 \pi / L$ (right).

\section{Summary and Conclusions}

We investigated a new method to compute nucleon form factors in lattice QCD based on spatially localizing the current with a Gaussian profile. We found evidence that for the transverse matrix elements of the axial current, excited states get additionally suppressed as the localization becomes stronger in position space, even though the dispersion relation of $G_{A}$ starts with a three-pion continuum. From the discussion around Eq. (2.5), we expect that for form factors whose dispersive representation starts with a two-pion cut (e.g. isovector vector or scalar isoscalar form factors), the excited-state contamination due to spatially distant contributions should be relatively more important, and it must be very large when the pion pole contributes. We were able to extract the axial charge and dipole-mass from fits to lattice data with a localized axial current in the plane transverse to the nucleon spin. In the near future, we will perform fits to several source-sink separations simultaneously, and apply the method to the extraction of $G_{P}$. Also, to better constrain the $Q^{2}$ dependence of the form factor, one could use as a set of wave packets the Hermite polynomials times a Gaussian of fixed width.

Acknowledgements: This project is supported by the DFG via the CRC 1044 and by the European Research Council (ERC) under the European Union's Horizon 2020 research and innovation programme through grant agreement 771971-SIMDAMA. The data has been produced on the HPC cluster "Clover" at the Helmholtz- 

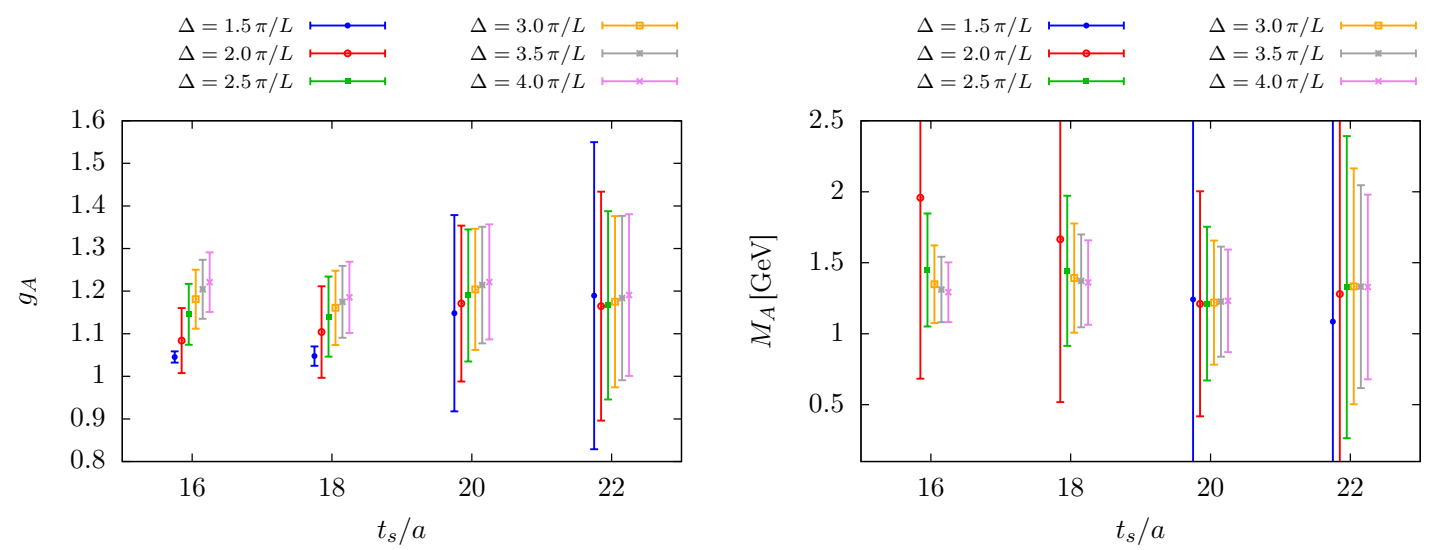

Figure 3: Summary of fit results for the axial charge (left) and the axial mass (right) for different momentumspace widths $\Delta$ of the wave packet.

Institute Mainz. The D200 ensemble was produced on JUQUEEN using computing time provided by the Gauss Centre for Supercomputing through the John von Neumann Institute for Computing.

\section{References}

[1] V. Bernard, L. Elouadrhiri and U.-G. Meißner, Axial structure of the nucleon, Journal of Physics G Nuclear Physics 28 (2002) R1 [hep-ph/0107088].

[2] J. Green, Systematics in nucleon matrix element calculations, in 36th International Symposium on Lattice Field Theory (Lattice 2018) East Lansing, MI, United States, July 22-28, 2018, 2018.

[3] O. Bär, Nucleon-pion-state contamination in lattice calculations of the axial form factors of the nucleon, in 36th International Symposium on Lattice Field Theory (Lattice 2018) East Lansing, MI, United States, July 22-28, 2018, 2018, 1808 .08738.

[4] K.-F. Liu, J. Liang and Y.-B. Yang, Variance Reduction and Cluster Decomposition, Phys. Rev. D97 (2018) 034507 [1705.06358].

[5] H. B. Meyer, Lorentz-covariant coordinate-space representation of the leading hadronic contribution to the anomalous magnetic moment of the muon, Eur. Phys. J. C77 (2017) 616 [1706.01139].

[6] M. Bruno, D. Djukanovic, G. P. Engel, A. Francis, G. Herdoiza, H. Horch et al., Simulation of QCD with $N_{f}=2+1$ flavors of non-perturbatively improved Wilson fermions, Journal of High Energy Physics 2 (2015) 43 [1411.3982].

[7] M. Lüscher and S. Schaefer, Lattice QCD without topology barriers, Journal of High Energy Physics 7 (2011) 36 [1105.4749].

[8] M. Bruno, T. Korzec and S. Schaefer, Setting the scale for the CLS $2+1$ flavor ensembles, Phys. Rev. D95 (2017) 074504 [1608.08900].

[9] G. S. Bali, S. Collins and A. Schaefer, Effective noise reduction techniques for disconnected loops in lattice qcd, Computer Physics Communications 181 (2010) 1570 .

[10] T. Blum, T. Izubuchi and E. Shintani, New class of variance-reduction techniques using lattice symmetries, Phys. Rev. D88 (2013) 094503 [1208 . 4349].

[11] K. Ottnad, T. Harris, H. Meyer, G. von Hippel, J. Wilhelm and H. Wittig, Nucleon charges and quark momentum fraction with $N_{f}=2+1$ Wilson fermions, ArXiv e-prints (2018) [1809.10638]. 